\title{
Mechanical Vibration Experiment of Superconducting Magnet ${ }^{*}$
}

\author{
Koichiro WAKI $^{* *}$, Hiroshi SEINO ${ }^{* *}$ and Ken NAGASHIMA ${ }^{* *}$ \\ ${ }^{* *}$ Cryogenic Systems, Maglev Systems Technology Division, Railway Technical Research Institute, \\ 2-8-38 Hikari-cho, Kokubunji-shi, Tokyo 185-8540, Japan \\ E-mail: waki@rtri.or.jp
}

\begin{abstract}
The resonance modes of the on-board superconducting magnet for the JR Maglev system actually obtained at the durability test were two (propulsion and levitation modes). To reduce the durability test period of the specimen by half, it was investigated in this study how those two resonance modes were simultaneously reproduced. It appears that those two resonance modes are simultaneously reproducible if the three accelerating points to reproduce its levitation mode are used, and if proper beams to shift the resonance frequencies of its propulsion and levitation modes are added.
\end{abstract}

Key words: Mechanical Vibration, Superconducting Magnet, Maglev System, Propulsion, Levitation, Resonance Mode, Durability, Simultaneous Reproduction

\section{Introduction}

In the JR Maglev system, vehicles are propelled, levitated and guided by magnetic interaction between electric currents flowing in superconducting coils (coils installed in on-board superconducting magnets) and ground coils (coils laid on ground). There are two kinds of ground coil; one is responsible for propelling the vehicle and named propulsion ground coil, and the other is responsible for levitating the vehicle and named levitation ground coil (see Fig.1.).

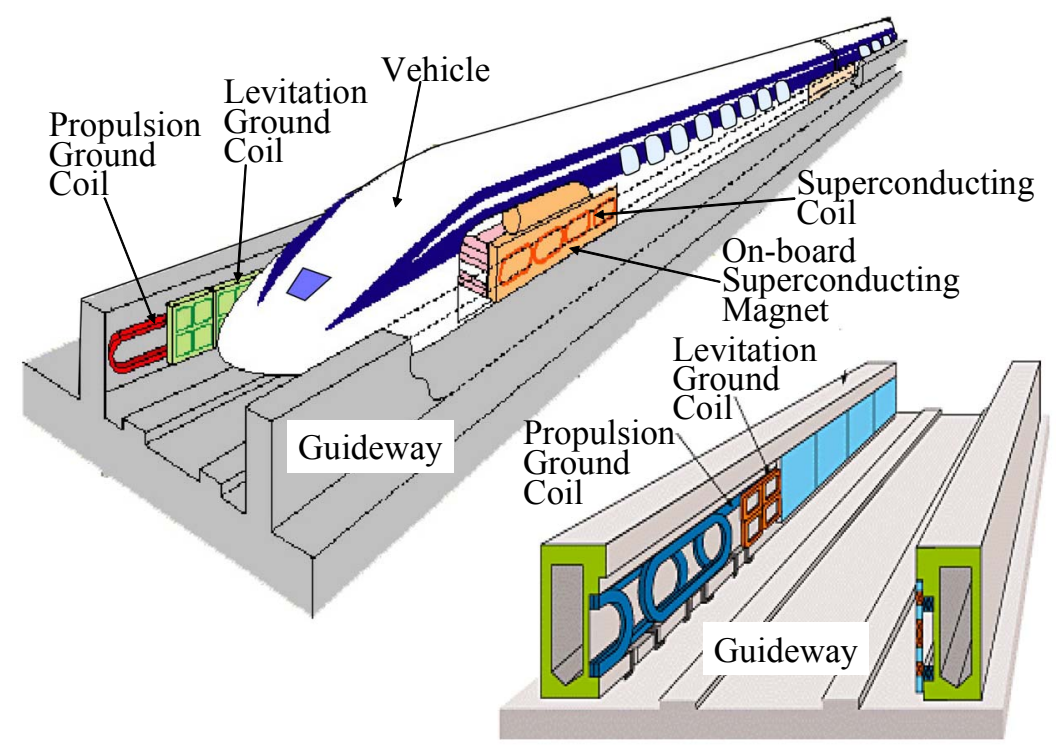

Fig.1 Arrangement of the superconducting coils and ground coils for the JR Maglev system

${ }^{*}$ Received 31 Oct., 2009 (No. 09-0659) [DOI: 10.1299/jmtl.3.187]

Copyright $\odot 2010$ by JSME 
The electric current flowing in the ground coils generates magnetic field which is synchronized with the vehicle running. Due to discrete arrangement of the ground coils, not only direct magnetic field but also alternating magnetic field is applied to the on-board superconducting magnets. Since alternating eddy current which is induced by the alternating magnetic field on surfaces of the on-board superconducting magnets and the electric current flowing in the superconducting coils magnetically interact, the on-board superconducting magnets are vibrated along with the vehicle running ${ }^{(1)-(3)}$.

One of the most important things for prospective commercial use of the on-board superconducting magnets is to prove their durability. However, the running test carried out on the Yamanashi Maglev Test Line had been insufficient regarding vibration resonance period if commercial use of fifteen years had been assumed. Therefore one of the on-board superconducting magnets was mechanically vibrated in a bench test during a period equivalent to the commercial use of fifteen years regarding the vibration resonance period. At that time mechanical vibration method under $\mathrm{LN}_{2}$ temperature excluding magnetic excitation was adopted to reproduce resonance mode of the on-board superconducting magnet because of its effectiveness (see Fig.2.). Thus the durability of the on-board superconducting magnets was proved for the commercial use ${ }^{(4)}$.

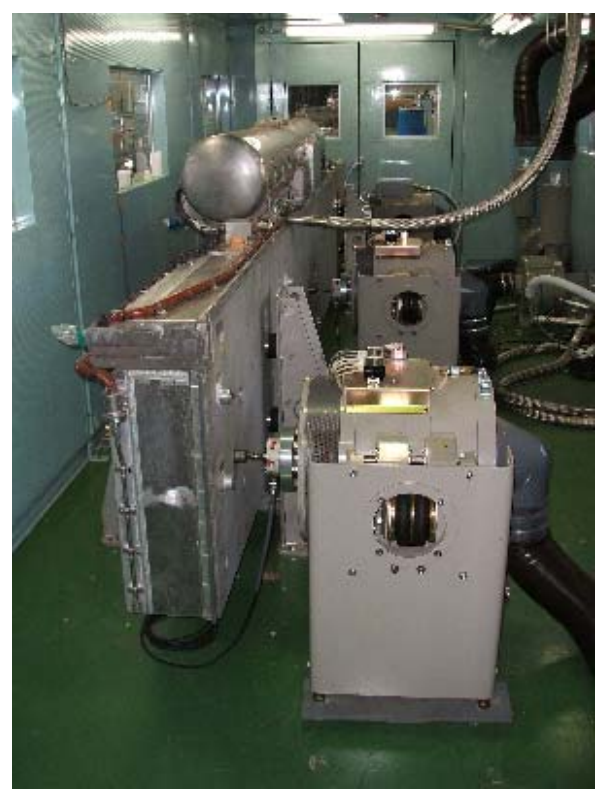

Fig.2 The durability test arrangement of the on-board superconducting magnet (on the left side) using three mechanical accelerators (on the right side)

The resonance modes of the on-board superconducting magnet actually obtained at the durability test were two; one was tertiary bend, corresponding to the propulsion ground coil and named propulsion mode, and the other was twist, corresponding to the levitation ground coil and named levitation mode (see Fig.3, one of the superconducting coils is drawn outside the on-board superconducting magnet as a matter of convenience.).

Figure 1 shows that height of the propulsion ground coils is close to height of the on-board superconducting magnets. Therefore the on-board superconducting magnets tend to yaw or be especially bent due to the propulsion ground coils. Figure 1 also shows that height of the levitation ground coils is close to height of the on-board superconducting magnets, and that the levitation ground coil consists of an upper part and a lower part whose magnetic polarity is (not shown in the figure but) reversed each other. Therefore the on-board superconducting magnets tend to roll or be especially twisted due to the levitation ground coils. 


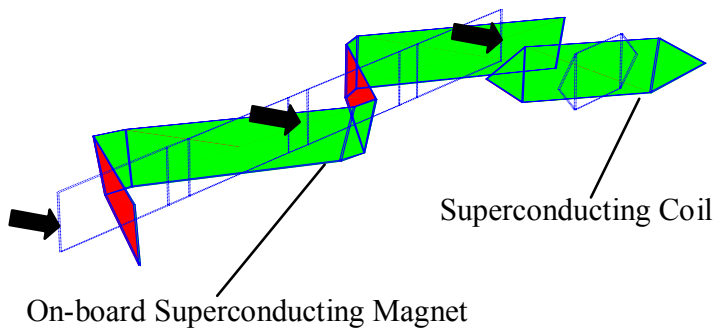

(a) Propulsion mode

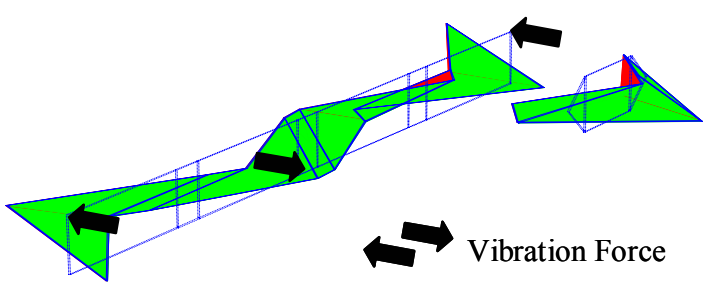

(b) Levitation mode

Fig.3 Resonance modes of the on-board superconducting magnet actually obtained at the durability test

Those two resonance modes were individually reproduced during the durability test of the on-board superconducting magnet ${ }^{(4)}$. If those two resonance modes were simultaneously reproduced, the durability test period of the specimen would be reduced by half. Hence it was investigated in this study how those two resonance modes were simultaneously reproduced.

\section{Condition to Simultaneous Reproduction Derived from Vibration Characteristics of the Specimen}

\subsection{Accelerating Points of the Specimen}

Since it is effective to accelerate an antinode of a specimen in order to reproduce its resonance mode, the accelerating points were individually selected and used to reproduce those two resonance modes during the durability test of the on-board superconducting magnet ${ }^{(4)}$ (see Fig.4.).

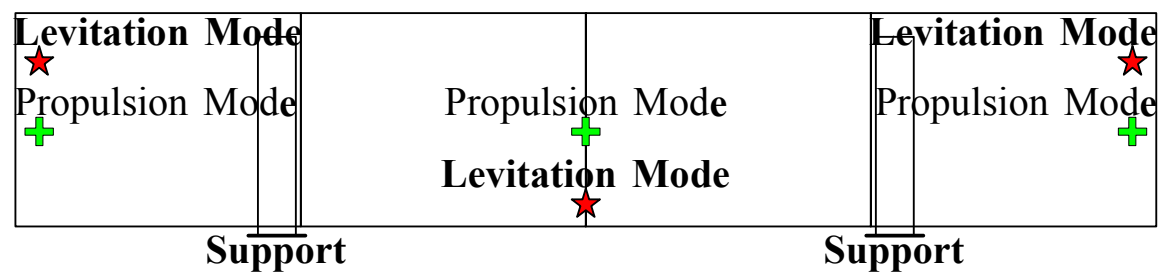

Fig.4 Accelerating points of the on-board superconducting magnet selected and used during the durability test

While the accelerating points to reproduce the propulsion mode of the on-board superconducting magnet were in a line located at the middle height of the specimen so as to be proper for tertiary bend, the accelerating points to reproduce the levitation mode of the on-board superconducting magnet were alternated in height of the specimen so as to be proper for twist. 
As for vibration force, while phases to reproduce the propulsion mode of the on-board superconducting magnet were in common so as to be proper for tertiary bend, phases to reproduce the levitation mode of the specimen were alternated so as to be proper for twist (see Fig.3.).

\subsection{Propulsion Mode Characteristics of the Specimen}

Since the superconducting coils of the on-board superconducting magnet are hardly deformed in case of the propulsion mode actually obtained at the durability test (see Fig.3.), resonance frequency is presumed to be single and dumping of vibration is presumed to be relatively small regarding the propulsion mode of the specimen. Actual results of the vibration test proved the above presumptions.

Due to the relatively small dumping of vibration, the propulsion mode of the on-board superconducting magnet will be reproduced if the specimen is accelerated at only one of its antinodes. Actually, when the specimen was accelerated at only one of the antinodes regarding the propulsion mode, the specimen was totally vibrated due to the small dumping of vibration (see Fig.5.).

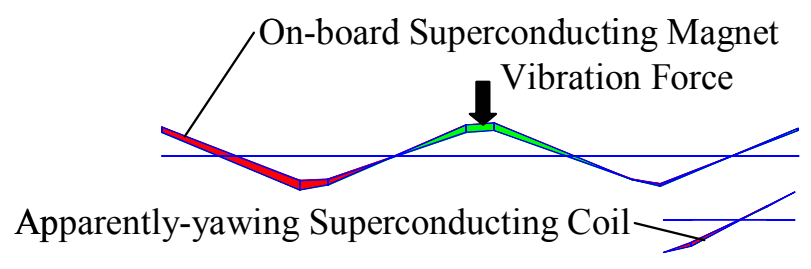

Fig.5 Top view of total vibration of the on-board superconducting magnet having undergone the durability test using a single accelerating point regarding the propulsion mode

However the accelerating points to be the antinodes regarding the propulsion mode of the on-board superconducting magnet should be at least three, because the single accelerator in our testing system cannot generate sufficiently large force.

\subsection{Levitation Mode Characteristics of the Specimen}

Since the superconducting coils of the on-board superconducting magnet are considerably twisted in case of the levitation mode actually obtained at the durability test (see Fig.3.), resonance frequency is presumed to be plural due to the slight difference between the superconducting coils and dumping of vibration is presumed to be relatively large regarding the levitation mode of the specimen. Actual results of the vibration test proved the above presumptions.

Due to the relatively large dumping of vibration, the levitation mode of the on-board superconducting magnet will not be reproduced unless the specimen is accelerated at three at least of its antinodes covering total length of the specimen. Actually, when the specimen was accelerated at only one of the antinodes regarding the levitation mode, the specimen was partially vibrated near the accelerating point due to the large dumping of vibration (see Fig.6.).

If the superconducting coil is twisted, mechanical heat is generated by friction between the superconducting coil and its fasteners ${ }^{(5)}$, and also the vibration is presumed to be dumped by the friction.

The three accelerating points to reproduce the levitation mode of the on-board superconducting magnet are also the antinodes regarding its propulsion mode. Therefore it appears that the propulsion and levitation modes of the specimen are simultaneously reproducible if the three accelerating points to reproduce its levitation mode are used. 


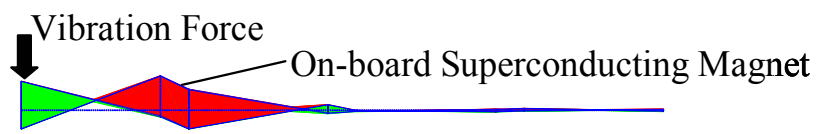

Slightly-twisting Superconducting Coil

(a) Left-end accelerating point

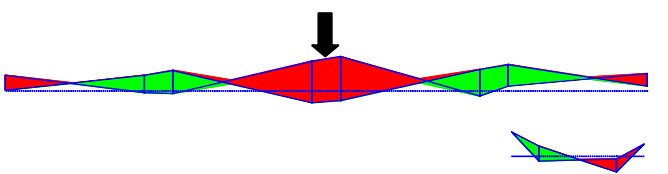

(b) Middle accelerating point

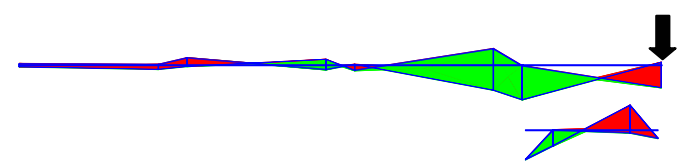

(c) Right-end accelerating point

Fig.6 Top view of partial vibration of the on-board superconducting magnet having undergone the durability test using a single accelerating point regarding the levitation mode

\section{Condition to Simultaneous Reproduction Derived from Functional Limits of the Accelerators}

\subsection{Additional Beams to the Specimen}

Actually resonance frequency regarding the levitation mode of the on-board superconducting magnet having undergone the durability test is higher than resonance frequency regarding the propulsion mode of the specimen. Therefore accelerators have to generate double frequencies of vibration force in order to simultaneously reproduce the propulsion and levitation modes of the specimen. The accelerators in our testing system can generate only harmonics of vibration force. Hence the propulsion and levitation modes of the specimen are simultaneously reproducible if resonance frequency ratio of its levitation mode to its propulsion mode is integer.

Since the resonance frequency ratio of the levitation mode to the propulsion mode of the on-board superconducting magnet had been larger than 2, the ratio was aimed at to be reduced to 2 with increasing the resonance frequency of its propulsion mode and with decreasing the resonance frequency of its levitation mode. According to structural dynamics analysis, frame thickness of the specimen may affect the resonance frequencies of its propulsion and levitation modes. Therefore proper beams to shift the resonance frequencies of its propulsion and levitation modes were planned to be added. It was desired but difficult that the beams satisfied the following all.

- In order to increase the resonance frequency of its propulsion mode, the beams are as long and as many as possible, covering the antinodes regarding its propulsion mode as many as possible.

- In order not to decrease the resonance frequency of its propulsion mode, the beams are as light and few as possible.

- In order not to increase the resonance frequency of its levitation mode, the beams are as close to the middle height of the specimen as possible, covering the nodes regarding its levitation mode.

- In order to decrease the resonance frequency of its levitation mode, the beams are as heavy and as many as possible. 
Figure 7 shows arrangement of the additional beams to the on-board superconducting magnet geometrically applicable to our testing system and actually prepared for a pretest. The beams were hollow pipes, and cross sectional shape of the beams was tetragon. The beams could be arranged at the right surface, the reverse surface, and both surfaces of the specimen.

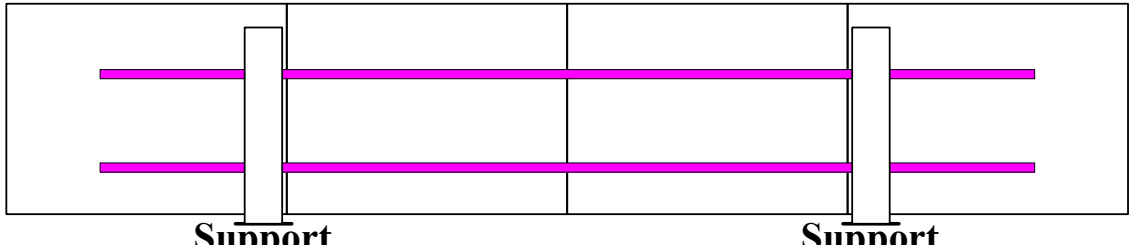

Support

Support

Fig.7 Arrangement of the additional beams to the on-board superconducting magnet geometrically applicable to our testing system and actually prepared for a pretest

Figure 8 shows an example of actual arrangement of the additional beams to the on-board superconducting magnet. The beams were arranged at the right surface of the specimen in this case.

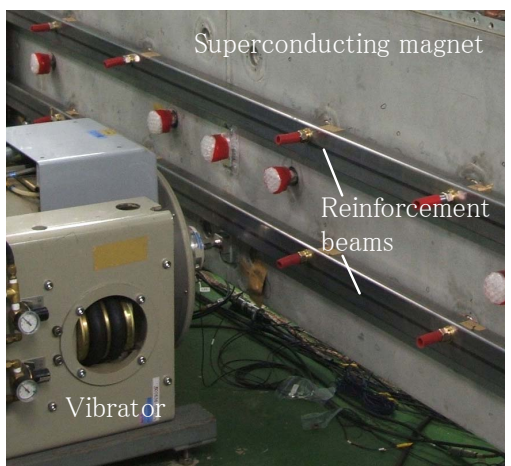

Fig.8 An example of actual arrangement of the additional beams to the on-board superconducting magnet

\subsection{Pretest Result of the Specimen}

In order to confirm effect of the additional beams, four cases of pretest was performed to the on-board superconducting magnet having undergone the durability test under room temperature because of its effectiveness as follows:

(A) without the additional beams

(B) with the additional beams arranged at the right surface of the specimen

(C) with the additional beams arranged at the reverse surface of the specimen

(D) with the additional beams arranged at both surfaces of the specimen

Figure 9 shows the pretest result of the on-board superconducting magnet with and without the additional beams regarding the resonance frequencies of its propulsion and levitation modes as follows:

(A) The resonance frequencies of its propulsion and levitation modes were used for normalization.

(B) Compared to (A), while the resonance frequency of its propulsion mode was hardly affected, the resonance frequency of its levitation mode was decreased. Since the right surface of the specimen is not so expected to undertake responsibility of its rigidity, the additional beams arranged at its right surface is presumed to be mainly additional weight resulting the decrement of the resonance frequency of its levitation mode. 
(C) Compared to (A), while the resonance frequency of its propulsion mode was increased, the resonance frequency of its levitation mode was hardly affected. Since the reverse surface of the specimen is so expected to undertake responsibility of its rigidity, the additional beams arranged at its reverse surface is presumed to be mainly additional reinforcement resulting the increment of the resonance frequency of its propulsion mode.

(D) Compared to (A), change of the resonance frequency was almost superposition of (B) and (C).

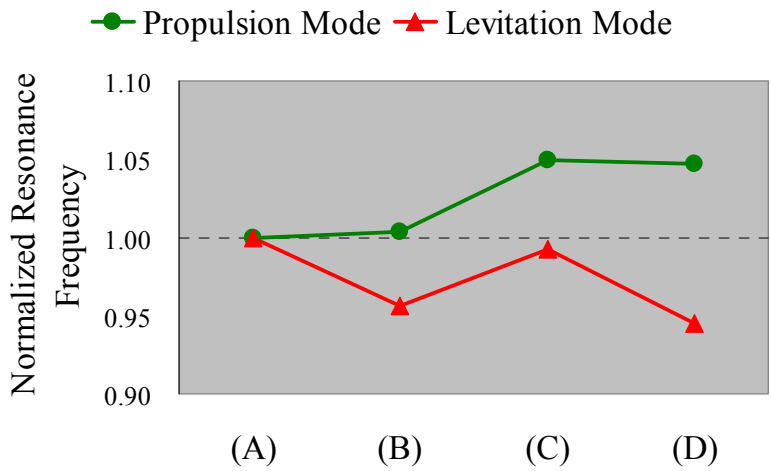

Fig.9 The pretest result of the on-board superconducting magnet with and without the additional beams regarding the resonance frequencies of its propulsion and levitation modes

Figure 10 shows the pretest result of the on-board superconducting magnet with and without the additional beams regarding the resonance frequency ratio of its levitation mode to its propulsion mode as follows:

(A) The resonance frequency ratio of its levitation mode to its propulsion mode was initially 2.43 .

(B) Compared to (A), the ratio was reduced to 2.31 since the resonance frequencies of its propulsion and levitation modes behaved as shown in Fig.9.

(C) Compared to (A), the ratio was reduced to 2.30 since the resonance frequencies of its propulsion and levitation modes behaved as shown in Fig.9.

(D) Compared to (A), the ratio was finally reduced to 2.19 since the resonance frequencies of its propulsion and levitation modes behaved as shown in Fig.9.

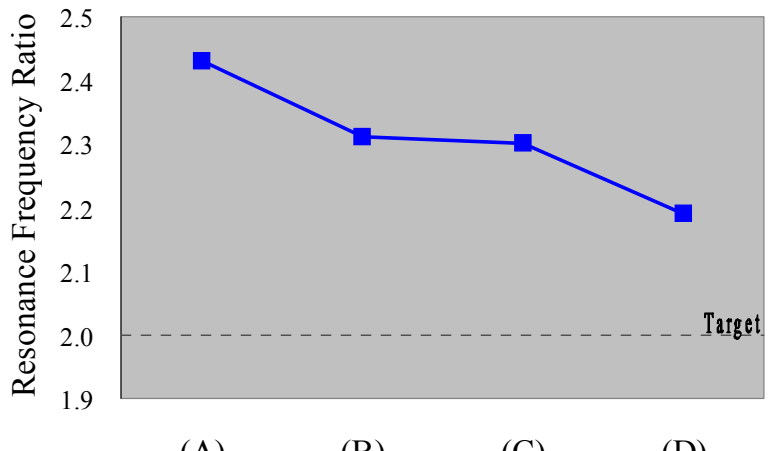

(A)

(B)

(C)

(D)

Fig.10 The pretest result of the on-board superconducting magnet with and without the additional beams regarding the resonance frequency ratio of its levitation mode to its propulsion mode 
Although the resonance frequency ratio of its levitation mode to its propulsion mode was not exactly reduced to 2 , it was confirmed that the additional beams were effective in the reduction of the ratio.

As for acceleration amplitude on vibration response of the on-board superconducting magnet, its changing ratio caused by the additional beams was less than $10 \%$. Therefore much larger vibration force will not be necessary, and no obstacle will happen regarding functional limits of the accelerators.

As for the vibration number of those two resonance modes of the on-board superconducting magnet, it was changed due to shifting the resonance frequencies with the additional beams. Therefore conversion will be necessary regarding the vibration number at the durability test.

\section{Conclusion}

The resonance modes of the on-board superconducting magnet for the JR Maglev system actually obtained at the durability test were two (propulsion and levitation modes). To reduce the durability test period of the specimen by half, it was investigated in this study how those two resonance modes were simultaneously reproduced.

Since the superconducting coils of the on-board superconducting magnet are hardly deformed in case of the propulsion mode, dumping of vibration is presumed to be relatively small regarding the propulsion mode of the specimen. Therefore the propulsion mode of the specimen will be reproduced if the specimen is accelerated at only one of its antinodes. However the accelerating points to be the antinodes regarding the propulsion mode of the specimen should be at least three, because the single accelerator in our testing system cannot generate sufficiently large force.

Since the superconducting coils of the on-board superconducting magnet are considerably twisted in case of the levitation mode, dumping of vibration is presumed to be relatively large regarding the levitation mode of the specimen. Therefore the levitation mode of the specimen will not be reproduced unless the specimen is accelerated at three at least of its antinodes covering total length of the specimen. And the three accelerating points to reproduce the levitation mode of the specimen are also the antinodes regarding its propulsion mode.

Resonance frequency regarding the levitation mode of the on-board superconducting magnet is higher than resonance frequency regarding the propulsion mode of the specimen. The accelerators in our testing system can generate only harmonics of vibration force. Since the resonance frequency ratio of the levitation mode to the propulsion mode of the specimen had been larger than 2, the ratio was aimed at to be reduced to 2. According to structural dynamics analysis, frame thickness of the specimen may affect the resonance frequencies of its propulsion and levitation modes. Therefore proper beams to shift the resonance frequencies of its propulsion and levitation modes were planned to be added.

In order to confirm effect of the additional beams, four cases of pretest was performed to the on-board superconducting magnet under room temperature because of its effectiveness. Although the resonance frequency ratio of its levitation mode to its propulsion mode was not exactly reduced to 2 , it was confirmed that the additional beams were effective in the reduction of the ratio.

Hence it appears that those two resonance modes are simultaneously reproducible if the three accelerating points to reproduce its levitation mode are used, and if proper beams to shift the resonance frequencies of its propulsion and levitation modes are added. 


\section{Acknowledgements}

Part of this study is financially supported by the Ministry of Land, Infrastructure, Transport and Tourism, Japan.

\section{References}

(1) Jizo, Y., Akagi, H., Yamaguchi, T., Terai, M., and Sinobu, M., Heat Load Characteristics and New Design Using One-coil Model Superconducting Magnets, Cryogenics, Vol. 37, No. 7 (1997), pp. 371-379.

(2) Ohmori, J., Nakao, H., Yamashita, T., Sanada, Y., Shudou. M., Kawai, M., Fujita, M., Terai, M., and Miura, A., Heat Load Tests of Superconducting Magnets Vibrated Electromagnetically for the Maglev Train, Cryogenics, Vol. 37, No. 8 (1997), pp. 403-407.

(3) Takizawa, T., Sonobe, T., Suzuki, F., Suzuki, E., Iwamatsu, M., Kishikawa, A., Terai, M., and Oishi, I., Development of a High Rigidity Superconducting Magnet for the Maglev System, Cryogenics, Vol. 37, No. 12 (1998), pp. 777-781.

(4) Inoue, A., Ogata, M., Nakauchi, M., Asahara, T., Herai, T., and Nishikawa, Y., Durability Evaluation of Superconducting Magnets, Journal of Physics, Conference Series 43 (2006), pp. 979-982, $7^{\text {th }}$ European Conference on Applied Superconductivity.

(5) Seino, H., Iwamatsu, M., Herai, T., Yoshino, Y., and Yamamoto, T., Dynamic Vibration Analysis and Tribological Study of Mechanical Heat Generation inside the Superconducting Coil for Maglev, JSME Int. J., Ser. C, Vol. 47, No. 2 (2004), pp. 457-463. 